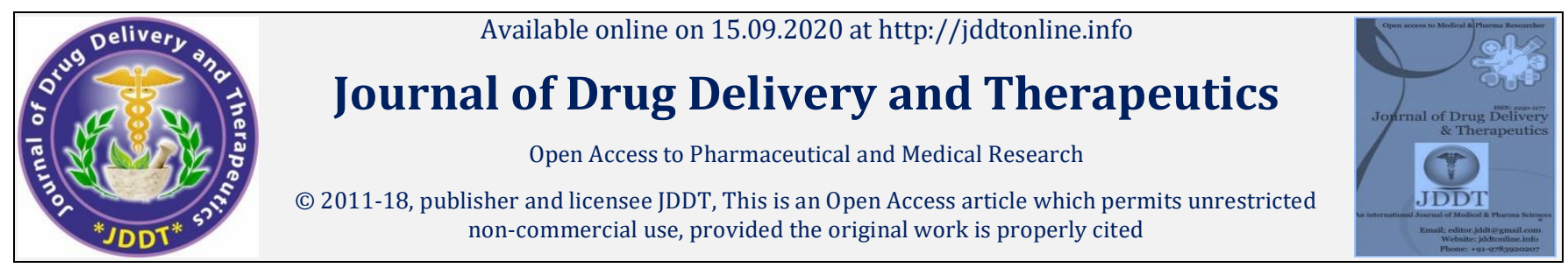

Open 8 Access

Review Article

\title{
A Laconic Review on Extraction, Biological Activities of Herbal Formulations of Berberine: A Traditional Drug
}

\author{
Sondhi Shivi ${ }^{1}$, Singh Navdeep ${ }^{1}$, Goyal Kamya ${ }^{2}$, Jindal Shammy ${ }^{1,3 *}$ \\ ${ }^{1}$ Department of Pharmaceutics, Laureate Institute of Pharmacy, Kathog, Jawalamukhi, Himachal Pradesh 176031, India \\ 2 Department of Pharmaceutical Chemistry and Analysis, Laureate Institute of Pharmacy, Kathog, Jawalamukhi, Himachal Pradesh 176031, India \\ ${ }^{3}$ Research scholar, Department of Pharmaceutics, Amity Institute of Pharmacy, Amity University Uttar Pradesh, Sector-125, Noida 201303, India
}

\begin{abstract}
Herbal formulation dosage form consists of one or more herbs processed in specified quantities to provide specific nutritional, cosmetic benefits use to diagnose the disease. Herbal formulations contain an active substance and preparation in combination of one or more herbal compounds. Berberis aristata is one of herbs of an ancient Ayurveda medicine and different properties along with various treatment of illness. Berberine, is a type of alkaloid which is quarternary protoberberine, is one of the known bioactive compounds scattered extensively in a number of clinically significant medicinal plants such as Hydrastis canadensis L., Phellodendron amurense R. ,Coptis japonica M., and Berberine containing plants have been used in traditional and folk medicine around the globe for centuries. It has been used for a, anti-pyretic diarrhea, bitter tonic, and eye infections. In the past three eras, Berberine has been studied intensively in over thousands cases because of its therapeutics, physicochemical effects, pharmacological, and physiological effects such as cardiovascular, anti-inflammatory, anti leshmanial, and anti- secretory, effects. Berberine act as a phytoconstituents in formulations and available in ayurveda, allopathy, and homeopathy medicines. With this review, we will evaluate the various traditional and medicinal use of Berberine and their isolation and extraction procedure. We will also review the potential of this plant as various dosage forms for the treatment of various diseases.
\end{abstract}

Keywords: Berberine; Extraction Method; Isolation method; Skin problems

Article Info: Received 10 July 2020; Review Completed 19 August 2020; Accepted 27 August 2020; Available online 15 Sep 2020

Cite this article as:

Sondhi S, Singh N, Goyal K, Jindal S, A Laconic Review on Extraction, Biological Activities of Herbal Formulations of Berberine: A Traditional Drug, Journal of Drug Delivery and Therapeutics. 2020; 10(5):345-357 http://dx.doi.org/10.22270/jddt.v10i5.4300

*Address for Correspondence:

Mr. Shammy Jindal, Department of Pharmaceutics, Laureate Institute of Pharmacy, Kathog, Jawalamukhi, Himachal Pradesh 176031, India

\section{INTRODUCTION}

Now-a-days herbal medicines are widely used and recommend for the treatment of patients health care. Herbal formulation dosage form consists of one or more herbs processed in specified quantities to provide specific nutritional, cosmetic benefits use to diagnose the disease. ${ }^{1}$ Herbal formulations contain an active substance and preparation in combination of one or more herbal compounds. Herbal formulations are obtained by extraction, distillation, expression, purification, fractionation include powdered of various crude plants. ${ }^{2}$

Herbal formulations are inexpensive, and possess good therapeutic action, better for patients' health care. Herbal formulations have no or less side effects as compared to allopathic or other systems. Herbal formulation is used in the treatment of medical conditions, enhancement of bioavailability, pharmacological activity and solubility. ${ }^{3}$
Ayurveda is a traditional system and improve physical, mental and emotional support for patients. Ayurveda is an ancient medicine healing system. It was developed more than 3000 years ago in India. Ayurveda helps to improve good health, cure disease, preventing and treating disease illness. Ayurveda believe in five basic elements found in universes such as space, air, fire, water and earth and human body systems supports three life energies or forces are known as doshas. Doshas are known as Vata dosha, Pitta dosha and Kapha dosha each controls in different body systems. ${ }^{3}$

Berberis aristata is one of herbs of an ancient Ayurveda medicine and different properties along with various treatment of illness. Berberine is an active phytoconstituents which is available in ayurveda, allopathy, and homeopathy medicines. The whole part of plant is also good source of dye and tannins. Berberine is main chemical constituents having various pharmacological actions. It is an active constituent 
benzylisoquinoline alkaloid and used in Ayurvedic and Chinese systems. ${ }^{4-5}$

This review evaluates various pharmacological traditional properties of Berberine along with their extractions method and potential use in the treatment of psoriasis and other disease.

\section{BASIC PROPERTIES AND USE OF BERBERINE}

The basic properties of Berberine were shown in Table 1. The chemical structure of Berberine is represented in Figure 1. Berberine has various biological and traditional uses which are listed in the Table 2 . Berberine was intensively used in the skin disorders and the whole information regarding their uses in skin disorders are listed in the Table 3.

Table 1: Basic properties of Berberine

\begin{tabular}{|l|l|l|}
\hline Sr. no. & \multicolumn{3}{|l|}{ Basic properties } \\
\hline $\mathbf{1}$ & Chemical formula & $\mathrm{C}_{20} \mathrm{H}_{18} \mathrm{NO}_{4}{ }^{+}$ \\
\hline $\mathbf{2}$ & Molecular mass & $336.366 \mathrm{gmol}^{-1}$ \\
\hline $\mathbf{3}$ & Structure name & $\begin{array}{l}5,6 \text {-dihydro-9,10-dimethoxybenzo[g]-1,3-benzodioxolo[5,6-a]quinolizinium (quaternary } \\
\text { amine) }\end{array}$ \\
\hline $\mathbf{4}$ & Basic nucleus & Quaternary benzylisoquinoline alkaloid molecule \\
\hline $\mathbf{5}$ & Nature & Non basic \\
\hline $\mathbf{6}$ & Appearance & Yellow solid \\
\hline $\mathbf{7}$ & Melting point & $145^{\circ} \mathrm{C}$ \\
\hline $\mathbf{8}$ & Solubility & Poor water soluble \\
\hline $\mathbf{9}$ & Salt & Water, acidic, neutral media \\
\hline $\mathbf{1 0}$ & Base & Soluble in organic solvents \\
\hline
\end{tabular}<smiles>COc1ccc2cc3[n+](cc2c1OC)CCc1c-3ccc2c1OCO2</smiles>

Figure 1: Berberine

Table 2: Biological and traditional uses of plants

\begin{tabular}{|c|c|c|c|}
\hline Sr. no. & Biological name & Plant name & Traditional uses \\
\hline 1 & Berberis vulgaris & Barberry & Acne, inflamed bumps, pimples, cardiovascular and hypertension \\
\hline 2 & Argemone mexicana & Prickly poppy & Malaria, jaundice, snake bites \\
\hline 3 & Berberis aristata & Turmeric & Antibacterial, anti-diarrheal and anticancer \\
\hline 4 & Mahonia aquifolium & Oregon grape & Eczema, tuberculosis, periodontitis, dysentery, wounds. \\
\hline 5 & Hydrastis canadensis & Goldenseal & $\begin{array}{l}\text { As astringent, bitter tonic, laxative, antidiabetic and muscular } \\
\text { stimulant }\end{array}$ \\
\hline 6 & Eschscholzia californica & Californian poppy & $\begin{array}{l}\text { Depression, nerve pain, psychiatric conditions, blood vessel } \\
\text { problems and sedation }\end{array}$ \\
\hline 7 & $\begin{array}{l}\text { Xanthorhiza } \\
\text { simplicissima }\end{array}$ & Yellow root & $\begin{array}{l}\text { Anti-inflammatory, astringent, antimicrobial, anticonvulsant and } \\
\text { immunostimulant }\end{array}$ \\
\hline 8 & Phellodendron amurense & Amur cork tree & $\begin{array}{l}\text { Osteoarthritis, weight loss and obesity, diarrhea, ulcers in stomach, } \\
\text { diabetes, pneumonia, anti-inflammatory activity }\end{array}$ \\
\hline 9 & Coptis chinensis & $\begin{array}{l}\text { Chinese } \\
\text { goldthread }\end{array}$ & Dye, wool and fibers \\
\hline 10 & Tinospora cordifolia & $\begin{array}{l}\text { Heart-leaved } \\
\text { moonseed }\end{array}$ & $\begin{array}{l}\text { Gout, lymphoma, rheumatoid arthritis, peptic ulcer, cancers and } \\
\text { immune system }\end{array}$ \\
\hline
\end{tabular}


Table 3: Berberine species uses in intestinal and skin disorder

\begin{tabular}{|c|c|c|c|}
\hline Berberine species & $\begin{array}{l}\text { References (work } \\
\text { done) }\end{array}$ & Berberine used in intestinal disorders & $\begin{array}{l}\text { Berberine used in } \\
\text { skin disorders }\end{array}$ \\
\hline $\begin{array}{l}\text { In the Berberidaceae family, } \\
\text { they used as raw material and } \\
\text { an important ingredient used } \\
\text { in Ayurvedic traditional } \\
\text { medicine and Chinese } \\
\text { medicine. Berberis genus } \\
\text { includes } 450-500 \text { species } \\
\text { which represent the most } \\
\text { natural supply of Berberine. } \\
\text { The plants which are related } \\
\text { genus are commonly used as } \\
\text { various activities. The } \\
\text { Berberine fruit (Berberine } \\
\text { vulgaris) is used as blood } \\
\text { purifying agent from the past } \\
\text { years. } \\
\text { Use of stem, root, bark of plant } \\
\text { rich in Berberine. Berberis } \\
\text { species history has quite } 3000 \\
\text { years. }\end{array}$ & $\begin{array}{l}\text { (Karimov, A. et al., } \\
\text { 1993, ; Birdsall, T.C. } \\
\text { et al., 1997; Gupta, A. } \\
\text { et al., 2014; Tandon, } \\
\text { R. et al., 2004 } \\
\text { Singh, A. et al., 2010; } \\
\text { Amritpal, S. et al., } \\
\text { 2010; Kulkarni, S.K. } \\
\text { et al., 2009)6-12 }\end{array}$ & $\begin{array}{l}\text { Treat hepatitis A, hepatitis B, hepatitis C } \\
\text { and hepatitis D, prostate cancer, syphilis, } \\
\text { poliomyelitis, conjunctivitis, leishmaniasis, } \\
\text { blood vomiting, jaundice, rheumatism, } \\
\text { body pains, sleeping sickness, treated } \\
\text { infectious disease such as urinary tract } \\
\text { infection, HIV sexual asthenia, diabetes, } \\
\text { hypertension, hemostatic, intestinal spams, } \\
\text { intestinal worms, fatigue, constipation, } \\
\text { dysentery, ulcer, intercostals pain, spleen } \\
\text { in children, sore, Treat various fevers: } \\
\text { yellow fever, rickettsia fever, typhoid } \\
\text { fever, chills, antipyretic, malaria, } \\
\text { Tuberculosis, coughs. }\end{array}$ & $\begin{array}{l}\text { In inflammation } \\
\text { condition, infectious } \\
\text { disorder on skin, } \\
\text { anti-diabetes, } \\
\text { aches, wounds, skin } \\
\text { ailments. }\end{array}$ \\
\hline
\end{tabular}

\section{EXTRACTION METHOD}

(Marek, R. et al., 2003; Grycova, L. et al., 2007) they reported that the Berberine are protoberberine alkaloid salts and they are changed in specific types of bases and extract further extracted in organic solvents. Berberine using classical extraction used different solvents e.g. acidified, aqueous mixtures, chloroform, ethanol and methanol. Sensitivity was major challenged for Berberine extraction and degrade Berberine light and also in case high temperature. ${ }^{13-14}$

(Babu, N.H.R. et al., 2012) they reported that they represent extraction process and drying temperature sample serious factor. Berberine found plant part of stem from Cosinium fenestratum and collect sample for the Berberine and extract yield from tissue sample was dried different types:

(i) Cold extraction method: in this method sample dried under refrigerator conditions $\left(-20^{\circ} \mathrm{C}\right)$ with methanol and ethanol. 15

(ii) Hot extraction method: in this method sample dried in water bath $\left(50^{\circ} \mathrm{C}\right)$ with ethanol and methanol.

Extract centrifuged and dried at constantly room temperature and filtered berberine sample analyzed. ${ }^{15}$

(Teng, H. et al.2013; Choi, Y. et al., 2013) they reported that the serious stage choice of solvents considered in Berberine. The solvents used mostly for the extraction process aqueous, acidified, methanol, ethanol and alkaloid rhizomes of coptis chinensis content Berberine extract. The acidified solvents (hydrochloride acid, phosphoric acid, nitric acid, sulfuric acid and acetic acid) used with combination higher solubility with free alkaloid base salts and concentration $0.34 \%$ phosphoric acid compared with classical extraction techniques. The extraction by the reflux and soxhlet method of Berberine at higher amount of yield contain by cold acid extraction. ${ }^{16}$

(Mokgadi, J.et al., 2013; Rojsanga, P.et al., 2005; Gritsanapan, W.et al., 2005) they reported that drawbacks of conventional methods and longtime extraction with large amount of solvents. They used maceration process procedure for the part of plant Coscinium fenestratumwith total $3200 \mathrm{ml}$ ethanol solvent (80\%) long time period. ${ }^{17-18}$

(Rojsanga, P.et al., 2006; Gritsanapan, W.et al., 2006) they reported that the used different extraction classical extraction as soxhlet, percolation and maceration techniques. The Berberine contain from stem part of plant Coscinium fenestratum lower amount as compared to previous study. The extract material used $30 \mathrm{~g}$ and solvents used $2000 \mathrm{ml}$ amount for maceration process, $600 \mathrm{ml}$ amount used for soxhlet extraction and $5000 \mathrm{ml}$ amount used for percolation extraction process. The extraction continued 7 days long time periods for the process maceration and soxhlet extraction process working 3 days. ${ }^{19}$

(Shigwan, H.et al., 2013) they reported that a large amount solvent was used in conventional hot extraction methods. The Berberine obtained stem bark from Berberis aristata and Berberis tinctoria was used $800 \mathrm{~g}$ with $2500 \mathrm{ml}$ methanol in temperature $50^{\circ} \mathrm{C}$ for $3 \mathrm{hr}$. The extraction method widely used traditional method to extract out of Berberine and variety method used for development. To improvement efficiency of extraction, decreased time consumed for extraction and amount of solvents used within extraction. Better results by used different extraction techniques found successfully. These methods are alternative techniques (MAE, PLE, SFE, UPE and USE) with compared with method of classical extraction. ${ }^{20}$

(Alupului, A.et al., 2009) they reported that ultrasonically extraction and microwave-assisted extraction was considered in efficient, simple method, and cheap cost techniques. ${ }^{21}$

(Chang, Y.et al., 2013) that was performed in lesser extraction time. In his procedure were used to selected combination ionic liquid, green solvents solutions and the extract Coptis chinesis contain Berberine by using USE method technique. ${ }^{22}$

(Xu, K.et al., 2018) they reported that the compared many extraction methods such as soxhlet extraction and distillation extraction collect phellodendrine to establish in proper manner high efficient method. The extraction of phellodendron fresh bark from Cortex phellodendri contains 
Berberine extract. In his case, Berberinein combination of solvents used methanol, ethanol, acidified solvents and water with the extraction contain higher amount of extraction yield. They was determined that Berberine extract use of hydrochloride acidified acid, methanol and USE technique much more efficient. They concluded that the higher yield extraction compared with soxhlet method and distillation method of Berberine. ${ }^{23}$

(Xi, J.et al., 2017) they reported that the classical extracted method technique was considered. This technique present many advantages increased the yield, extraction time reduce, quality enhanced and reduced solvent consumption. ${ }^{24}$

(Guoping, L. et al., 2012) they reported that the Cortex phellodendri made comparison with different extraction techniques; heat reflux, MAE, UPE and USE techniques. The yield was obtained by extraction method in case UPE with lower extraction time and higher extraction time. Reflux, MAE and USE with $5.35 \mathrm{mg} / \mathrm{g}$ for $2 \mathrm{hrs}, 5.61 \mathrm{mg} / \mathrm{g}$ for $1 \mathrm{hrs}$ and $6 \mathrm{mg} / \mathrm{g}$ for $15 \mathrm{~min}^{25}$

(Mustafa, A.et al., 2011 ;Turner, C.et al., 2011) they reported that the considered both extraction method pressurized method and other method used accelerated solvent extraction. The plant extraction used as a technology for compound to pressurized liquid extraction and pressurized fluid extraction. ${ }^{26}$ The various methods of extraction of Berberine are listed in the Table 4.

Table 4: Various methods of extraction of Berberine from various crude drugs

\begin{tabular}{|c|c|c|c|c|}
\hline $\begin{array}{l}\text { Sr. } \\
\text { no. }\end{array}$ & Crude drug & References & Method extraction and Isolation & $\begin{array}{l}\text { Method of analysis various } \\
\text { analytical techniques }\end{array}$ \\
\hline 1 & $\begin{array}{l}\text { Argemone } \\
\text { mexicana }\end{array}$ & $\begin{array}{l}\text { (Samal, P.K. et } \\
\text { al.,2013) }\end{array}$ & $\begin{array}{l}\text { Method- Soxhlet extraction } \\
\text { Solvents: methanol } \\
\text { Dry the compound by evaporation } \\
\text { and } \\
\text { Re-solubilized the dried compound } \\
\text { in methanol with required } \\
\text { concentration. }\end{array}$ & $\begin{array}{l}\text { Method- HPTLC } \\
\text { Stationary Phase: silica gel } \\
\text { (60F254) } \\
\text { Mobile phase: Toluene: Ethyl } \\
\text { acetate (9:3, v/v). } \\
\text { Detection of compound at : } 266 \mathrm{~nm}\end{array}$ \\
\hline 2 & $\begin{array}{l}\text { Berberis aristata } \\
\text { DC } 1.5 \mathrm{~g} \text { and } \\
\text { herb extract } 0.1 \\
\mathrm{~g}\end{array}$ & $\begin{array}{l}\text { (Singh, R. et } \\
\text { al.,2010) }\end{array}$ & $\begin{array}{l}\text { Method- Reflux isolation } \\
\text { Solvents for extraction: Take } 100 \mathrm{~mL} \\
\text { methanol on a water bath for } 1 \mathrm{hr} \text {. } \\
\text { Filter process- } \\
\text { Re-isolate the crude by use of ES in } \\
50 \text { ml volume and repeat the } \\
\text { procedure } 2 \text { times for } 30 \text { min. } \\
\text { Filtrates combination and } \\
\text { concentration to } 50 \mathrm{ml} \\
\text { Herb extracts Method- ultrasonic } \\
\text { extraction } \\
\text { Solvents: Methanol } \\
\text { Volume: } 10 \text { mL approx. } \\
\text { Sonication process and Filtration } \\
\text { process is done. }\end{array}$ & $\begin{array}{l}\text { Method- HPLC Column: Zorbax ODS } \\
\text { II, } 250 \text { x } 4.6 \mathrm{~mm}(5 \mu \mathrm{m}) \text { Mobile } \\
\text { phase: potassium hydrogen } \\
\text { phosphate buffer } \\
\text { (pH 2.5). } \\
\text { Detection at: } 346 \mathrm{~nm} \\
\text { Temperature: } 40^{\circ} \mathrm{C} \\
\text { Flow Rate: } 1 \mathrm{ml} / \mathrm{min} \\
\text { Method- Herbal ultrasonic } \\
\text { extraction process } \\
\text { Solvents used: methanol (Volume } \\
\text { 25ml minimum) } \\
\text { Sonication process. }\end{array}$ \\
\hline 3 & $\begin{array}{l}\text { Berberis } \\
\text { aristata, } \\
\text { Berberis } \\
\text { tinctoria }\end{array}$ & $\begin{array}{l}\text { (Shigwan, H.et } \\
\text { al., 2013) }\end{array}$ & $\begin{array}{l}\text { Method: Hot extraction process } \\
\text { Solvents Used: methanol (2.5 Litre) } \\
\text { Time: Min. } 3 \mathrm{hr} \\
\text { Temperature: } 50^{\circ} \mathrm{C}\end{array}$ & $\begin{array}{l}\text { Method- HPLC } \\
\text { Column: Unisphere is used (C18, } \\
150 \times 4.6 \mathrm{~mm} \text { or } 5 \mu \mathrm{m}) \\
\text { Mobile Phase: } \\
0.1 \% \text { (trifluoroacetic acid) } \\
\text { Acetonitrile in the ratio of } \\
(60: 40 \mathrm{v} / \mathrm{v}) \\
\text { Detection at: } 350 \mathrm{~nm} \\
\text { Temperature: } 30^{\circ} \mathrm{C} \\
\text { Flow Rate: } 1 \mathrm{ml} / \mathrm{min}\end{array}$ \\
\hline 4 & Coptis chinensis & $\begin{array}{l}\text { (Teng, H, Choi, } \\
\text { Y.et al., 2013)16 }\end{array}$ & $\begin{array}{l}\text { Method- Acid assisted extraction } \\
\text { process. }\end{array}$ & $\begin{array}{l}\text { Method- HPLC Column used: XTerra } \\
(\mathrm{C} 18,250 \times 4.6 \mathrm{~mm})\end{array}$ \\
\hline
\end{tabular}




\begin{tabular}{|c|c|c|c|c|}
\hline & & & $\begin{array}{l}\text { Solvents used: Inorganic acids eg: } \\
\text { HCL } \\
\text { Phosphoric acid, nitric acid, and } \\
\text { sulfuric acid) and one } \\
\text { Organic acid eg: Acetic acid } \\
\text { Time: } 1 \text { to } 8 \mathrm{hr} \text {, } \\
\text { Concentrations Range of acid: } 0 \\
\text { to } 1 \% \\
\text { Ratios: } 20 \text { to } 60 \mathrm{~mL} / \mathrm{g} \\
\text { Maceration temp.: } 25^{\circ} \mathrm{C} \\
\text { Filtration: Final volume is diluted } \\
\text { with } 100 \mathrm{~mL} \\
\text { Method- Soxhlet extraction process } \\
\text { Solvents used: ethanol } \\
\text { Time: } 4 \text { hr } \\
\text { Temperature: } 70^{\circ} \mathrm{C} \\
\text { Dry the extract by evaporation and } \\
\text { Re-solubilized in } 100 \mathrm{ml} \\
\text { Method- Heating reflux extraction } \\
\text { process } \\
\text { Solvents used: ethanol } \\
\text { Soaking time: } 1 \text { hr } \\
\text { Extraction time: } 4 \text { hr } \\
\text { Temperature: } 70^{\circ} \mathrm{C} \text { on water bath } \\
\text { Filtration: Dilute with } 100 \text { mL. }\end{array}$ & $\begin{array}{l}\text { Mobile phase used: } \\
\text { Acetonitrile } \\
\text { Potassium dihydrogen phosphate } \\
\text { Ratio: }(27: 75 \mathrm{v} / \mathrm{v}) \\
\text { Detection at: } 345 \mathrm{~nm} \\
\text { Temperature: } 30^{\circ} \mathrm{C}\end{array}$ \\
\hline 5 & $\begin{array}{l}\text { Cortex } \\
\text { phellodendri }\end{array}$ & $\begin{array}{l}\text { (Guoping, L.et } \\
\text { al., 2012) }\end{array}$ & $\begin{array}{l}\text { Method- Ultrahigh pressure } \\
\text { extraction (UPE) } \\
\text { Parameters used: } \\
\text { Solvents used: ethanol } \\
\text { Ratio of liquid-solid: } 31.3 \\
\text { Pressure of extraction: } 243.30 \\
\text { Time: } 2 \text { min }\end{array}$ & $\begin{array}{l}\text { Method- HPLC } \\
\text { Column Used: Hypersil Oxidative } \\
\text { Desulfurization (C18, } 250 \times 4.6 \mathrm{~mm} \\
\text { and } 5 \mu \mathrm{m}) \\
\text { Mobile Phases used: } \\
\text { triethanolamine } \\
\text { Solution pH: } 3.5 \\
\text { Detection at: } 265 \mathrm{~nm} \\
\text { Temperature: } 30^{\circ} \mathrm{C} \\
\text { Flow Rate: } 1 \mathrm{ml} / \mathrm{min}\end{array}$ \\
\hline 6 & Coptis rhizome & $\begin{array}{l}\text { Liu, B.et } \\
\text { al.,2006) }\end{array}$ & $\begin{array}{l}\text { Method: Supercritical fluid extraction } \\
\text { process } \\
\text { Time: More than } 3 \mathrm{hr} \\
\text { Temperature: } 60^{\circ} \mathrm{C} \\
\text { Pressure range: } 200-500 \mathrm{bar} \\
\text { Flow-rate of CO: } 1 \text { Litre/min } \\
\text { Flow-rate of modifier system: } 0.4 \\
\text { mL/min. } \\
\text { Organic solvent: } \\
\text { ethanol } \\
\text { carbon dioxide, } \\
\text { methanol }\end{array}$ & $\begin{array}{l}\text { Methods: HPLC } \\
\text { Column Used: Diamonsil (C18, } 250 \\
\times 4.6 \mathrm{~mm} \text { and } 5 \mu \mathrm{m}) \\
\text { Mobile Phases used: } \\
\text { Potassium di-hydrogen phosphate: } \\
\text { Acetonitrile } \\
\text { Detection at: } 345 \mathrm{~nm} \\
\text { Flow rate: } 1 \mathrm{ml} / \mathrm{min}\end{array}$ \\
\hline
\end{tabular}




\begin{tabular}{|c|c|c|c|c|}
\hline & & & $\begin{array}{l}\text { Tween } \\
\text { Soxhlet extraction: } \\
\text { Extraction Solvents: hydrochloric } \\
\text { acid: methanol }(1: 100, \mathrm{v}) \\
\text { Time: } 8 \mathrm{~h}\end{array}$ & \\
\hline 7 & $\begin{array}{l}\text { Cortex } \\
\text { pellodendri } \\
\text { Amurensis }\end{array}$ & $\begin{array}{l}\text { (Liu, S.et al., } \\
\text { 2013)30 }\end{array}$ & $\begin{array}{l}\text { Method: Ultrahigh pressure } \\
\text { extraction } \\
\text { Solvents used: ethanol } \\
\text { Ratio of liquid-solid : } 30: 1 \\
\text { Extracting pressure : } 400 \mathrm{MPa} \\
\text { Time: } 4 \mathrm{~min} \\
\text { Temperature : } 40^{\circ} \mathrm{C} \\
\text { Method: Ultrasonic extraction } \\
\text { process } \\
\text { Solvents used: ethanol } \\
\text { Soaking time for sample: } 24 \mathrm{hr} \\
\text { Sonication time: } 60 \text { min } \\
\text { Temperature: } 30^{\circ} \mathrm{C} \\
\text { Method- Heat reflux extraction } \\
\text { process } \\
\text { Solvent used: ethanol } \\
\text { Soaking time for sample: } 24 \mathrm{hr} \\
\text { Extraction time: } 4 \mathrm{hr} \\
\text { Method- Soxhlet extraction process } \\
\text { Solvent used: ethanol } \\
\text { Soaking time: } 4 \text { hr Time of sample } \\
\text { isolation: } 4 \text { hr }\end{array}$ & $\begin{array}{l}\text { Method: HPLC } \\
\text { Column used: Daisopak (SP-120-5- } \\
\text { ODS_BP, } 250 \times 4.6 \mathrm{~mm} \text { and } 5 \mu \mathrm{m}) \\
\text { Mobile Phases used: } \\
\text { Acetonitrile } \\
\text { phosphoric acid } \\
\text { water ratio: }[0.7: 100 \mathrm{v} / \mathrm{v}] \\
\text { Detection at: } 345 \mathrm{~nm} \\
\text { Temperature: } 25^{\circ} \mathrm{C} \\
\text { Flow Rate: } 1 \mathrm{~mL} / \mathrm{min}\end{array}$ \\
\hline 8 & $\begin{array}{l}\text { Coscinium } \\
\text { fenestratum }\end{array}$ & $\begin{array}{l}\text { (Rojsanga, P.et } \\
\text { al.,2005; } \\
\text { Gritsanapan, } \\
\text { W.et al., 2005)18 }\end{array}$ & $\begin{array}{l}\text { Method: Maceration } \\
\text { Extraction process } \\
\text { Solvents used: ethanol } \\
\text { Shaking time: } 80 \mathrm{hr} \text { rpm-200 } \\
\text { Re-extraction time: } 48 \mathrm{hr} \\
\text { Shaking time: } 24 \mathrm{hr} \\
\text { Combination of extracts } \\
\text { concentration } \\
\text { Dryness: By evaporation) } \\
\text { Re-solubilized: } 10 \text { mg dried extract } \\
\text { in ethanol }\end{array}$ & $\begin{array}{l}\text { Method: TLC } \\
\text { Stationary phase used: Silica gel } \\
\text { (GF254) } \\
\text { Mobile phase used: } \\
\text { ethyl acetate: butanol: formic acid } \\
\text { :water (50:30:12:10) } \\
\text { Detection at: } 366 \mathrm{~nm}\end{array}$ \\
\hline 9 & $\begin{array}{l}\text { Coscinium } \\
\text { fenestratum }\end{array}$ & $\begin{array}{l}\text { (Arawwawala, } \\
\text { L.D.et al.,2012; } \\
\text { Wickramaar, } \\
\text { W.A.et } \\
\text { al.,2012)31 }\end{array}$ & $\begin{array}{l}\text { Solvent used: methanol } \\
\text { Extraction (Hot): sample refluxed } \\
\text { with solvent upto3 hr } \\
\text { Filtration: Methanol } \\
\text { Evaporation: Methanol. } \\
\text { Extracts Re-solubilized in: Methanol } \\
\text { Method: Cold extraction process } \\
\text { Solvent used: ES } \\
\text { Time: } 24 \text { hr } \\
\text { Filtration/evaporation }\end{array}$ & $\begin{array}{l}\text { Method: TLC } \\
\text { Adsorbent used: Silica Gel (GF-254) } \\
\text { Solvent used: n-Butanol: Ethyl } \\
\text { acetate: Acetic acid } \\
\text { Ratio: }(2.5: 1.5: 1, \mathrm{v} / \mathrm{v} / \mathrm{v}) \\
\text { Detection at: } 254 \text { and } 366 \mathrm{~nm}\end{array}$ \\
\hline
\end{tabular}




\begin{tabular}{|c|c|c|c|c|}
\hline & & & /Resolubilization: Methanol & \\
\hline 10 & $\begin{array}{l}\text { Dried powder } \\
\text { stem Coscinium } \\
\text { fenestratum }\end{array}$ & $\begin{array}{l}\text { (Akowuah, } \\
\text { G.A.et al.,2014; } \\
\text { Rojsanga, P. et } \\
\text { al., 2010) }\end{array}$ & $\begin{array}{l}\text { Solvents used: water/ } \\
\text { methanol/water }(1: 1: 1 \mathrm{v} / \mathrm{v}) \\
\text { Methanol Sonication time: } 15 \\
\text { minTemperature: room temp. } \\
\text { Centrifugation process: } 2800 \mathrm{rpm} \text { for } \\
\text { 15min. } \\
\text { Filtration/evaporation/ } \\
\text { Resolubilization: Methanol:water } \\
\text { Ratio: } 9: 1 \mathrm{v} / \mathrm{v}\end{array}$ & $\begin{array}{l}\text { Method- HPLC Column used: } \\
\text { (Oxidative Desulfurization, } \\
\text { Chromolith, RP-18e,100 × } 4.6 \mathrm{~mm} \text { ) } \\
\text { Mobile Phases used: } \\
\text { Methanol } \\
\text { Deionized } \\
\text { Ratio: Water }(90: 10 \mathrm{v} / \mathrm{v}) \\
\text { Flow rate: } 0.5 \mathrm{~mL} / \mathrm{min} \\
\text { Temperature: } 25^{\circ} \mathrm{C} \text { Detection: UV }\end{array}$ \\
\hline 11 & $\begin{array}{l}\text { Dried form } \\
\text { Coscinium } \\
\text { fenestratum }\end{array}$ & $\begin{array}{l}\text { (Babu, N.H.R.et } \\
\text { al.,2012) }\end{array}$ & $\begin{array}{l}\text { Method- Cold extraction process } \\
\text { Temperature: } 20^{\circ} \mathrm{C} \\
\text { Hot extraction temperature: } 50^{\circ} \mathrm{C} \text { on } \\
\text { water bath } \\
\text { Solvent used: ethanol } \\
\text { Centrifugation time: } 10 \text { min } \\
\text { Temperature: } 10^{\circ} \mathrm{C} \text { Samples }\end{array}$ & $\begin{array}{l}\text { Method: HPLC Column used: (C18, } \\
250 \times 4.6 \mathrm{~mm} \text { and } 5 \mu \mathrm{m}) \\
\text { Mobile Phases used: } \\
\text { Acetonitrile } \\
\text { Trifluro-acetic acid } \\
\text { Ratio ( } 50: 50, \mathrm{v} / \mathrm{v}) \\
\text { Detection at: } 344 \mathrm{~nm} \\
\text { Flow rate: } 0.8 \mathrm{ml} / \mathrm{min}\end{array}$ \\
\hline 12 & $\begin{array}{l}\text { Goldenseal, } \\
\text { Hydrastis } \\
\text { canadensis }\end{array}$ & $\begin{array}{l}\text { (Mokgadi, J.et } \\
\text { al., 2013) }\end{array}$ & $\begin{array}{l}\text { Method: Pressurized hot water } \\
\text { extraction process } \\
\text { Solvent used: water } \\
\text { Temperature: } 140^{\circ} \mathrm{C} \\
\text { Parameters used: } \\
\text { Pressure: } 50 \text { bars } \\
\text { Flow rate: } 1 \mathrm{~mL} / \mathrm{min} \\
\text { Time: } 15 \mathrm{~min} \\
\text { Method: Reflux extraction process } \\
\text { Solvent used: methanol } \\
\text { Sonication time: } 4 \text { hr } \\
\text { Sonication temperature: } 80^{\circ} \mathrm{C} \\
\text { Method: Ultrasonic extraction } \\
\text { process } \\
\text { Solvent used: Methanol } \\
\text { Reflux time: } 6 \text { hr by continuously } \\
\text { stirring process. }\end{array}$ & $\begin{array}{l}\text { Method: HPLC (Diode Array Diode) } \\
\text { Column used: Zorbax eclipse Plus (C } \\
\text { 18, } 75 \times 4.6 \mathrm{~mm} \text { and } 3.5 \mu \mathrm{m} \text { ) } \\
\text { Mobile phase used: } \\
\text { Formic Acid- pH } 2.7 \\
\text { Methanol } \\
\text { Detection at: } 242 \mathrm{~nm} \\
\text { Temperature: } 35^{\circ} \mathrm{C} \\
\text { Flow rate: } 1 \mathrm{ml} / \mathrm{min} \\
\text { MS } \\
\text { Detection by: Elect spray ionization } \\
\text { (+) } \\
\text { Temperature of capillary: } 200^{\circ} \mathrm{C} \\
\text { Current: } 20 \mathrm{~V} \\
\text { Voltage of tube lens: } 5 \mathrm{~V}\end{array}$ \\
\hline 13 & $\begin{array}{l}\text { Root Berberis } \\
\text { aristata DC }\end{array}$ & $\begin{array}{l}\text { (Patel, M.C.et } \\
\text { al.,2013) }\end{array}$ & $\begin{array}{l}\text { Method: Soxhlet extraction process } \\
\text { Solvent used: Ethanol is used to form } \\
\text { an extract. } \\
\text { Desolvation: hot water filtration: hot } \\
\text { water } \\
\text { Acidification: HCL } \\
\text { Cool at: ice bath } \\
\text { Cooling time: } 30 \mathrm{~min} \\
\text { Eg: overnight in a refrigerator }\end{array}$ & $\begin{array}{l}\text { Method: HPTLC } \\
\text { Stationary phase used: Pre-coated } \\
\text { silica gel Grade: } 60 \mathrm{GF}-254 \\
\text { Mobile phases used: } \mathrm{n} \text {-butanol and } \\
\text { glacial acetic acid or water } \\
\text { Ratio: }(12: 3: 4 \mathrm{v} / \mathrm{v} / \mathrm{v}) \\
\text { Temperature: } 33 \pm 5^{\circ} \mathrm{C} \\
\text { Detection at: } 350 \mathrm{~nm}\end{array}$ \\
\hline 14 & Rabbit plasma & $\begin{array}{l}\text { (Liu, M.et al., } \\
\text { 2011)35 }\end{array}$ & $\begin{array}{l}\text { Mix } 100 \mu \mathrm{l} \text { of sample in } 3 \% \text { of formic } \\
\text { acid with Acetonitrile solvent } \\
\text { Solvent Volume: }(200 \mu \mathrm{l}) \\
\text { Vortex time: } 30 \mathrm{sec}\end{array}$ & $\begin{array}{l}\text { Method: } \\
\text { Liquid Chromatography- } \\
\text { Electrospray Ionization }\end{array}$ \\
\hline
\end{tabular}




\begin{tabular}{|c|c|c|c|c|}
\hline & & & $\begin{array}{l}\text { Centrifugation time: } 10 \mathrm{~min} \\
\text { Centrifugation temperature: } 4^{\circ} \mathrm{C} \\
\text { Evaporation: nitrogen stream } \\
\text { Evaporation temperature: } 40^{\circ} \mathrm{C} \\
\text { Solubilization process: } 100 \mu \mathrm{l} \text { with } \\
(20 \% \text { methanol) }\end{array}$ & $\begin{array}{l}\text { Mass Spectroscopy } \\
\text { HPLC } \\
\text { Column used: Capcell Pakc18 (MG, } \\
100 \times 2.1 \mathrm{~mm} \text { and } 5 \mu \mathrm{m}) \text { Mobile } \\
\text { Phases: } \\
\text { formic acid } \\
\text { methanol } \\
\text { Ratio: }(60: 40 \mathrm{v} / \mathrm{v}) \\
\text { Temperature: } 25^{\circ} \mathrm{C} \\
\text { Flow rate: } 0.4 \mathrm{ml} / \mathrm{min} \\
\text { Method- Mass Spectroscopy } \\
\text { detection process } \\
\text { Source: Electro-spray Ionization } \\
\text { Quantification mode: MRM }\end{array}$ \\
\hline 15 & $\begin{array}{l}\text { Rat plasma } \\
\text { Rat tissue }\end{array}$ & $\begin{array}{l}\text { (Wang, Z.et } \\
\text { al.,2016) }\end{array}$ & $\begin{array}{l}\text { Method- Rat plasma process } \\
\text { Solvent used: methanol } \\
\text { Mixing ratio: } 200 \mu \text { l with standard } 40 \\
\mu \text { l and } 560 \mu \text { solvent. } \\
\text { Vortex at: } 20 \text { sec } \\
\text { Centrifugation time: } 10 \mathrm{~min} \text { rpm: } \\
12000 \\
\text { Filtration process: Rat tissue } \\
\text { Grinding process: } 3 \mathrm{ml} \text { saline solution } \\
\text { with } 600 \text { mg tissue } \\
\text { Centrifugation time: } 10 \mathrm{~min} \text { rpm: } \\
12000 \\
\text { Mixing ratio: } 200 \mu \mathrm{l} \text { with standard } 40 \\
\mu l \text { in } 560 \mu \mathrm{l} \\
\text { Vortex at: } 20 \text { sec } \\
\text { Centrifugation time: } 10 \mathrm{~min} \text { rpm: } \\
12000\end{array}$ & $\begin{array}{l}\text { Method: Ultra Performance Liquid } \\
\text { Chromatography (UPLC) or Mass } \\
\text { Spectroscopy process } \\
\text { Column used: Acquity Ethylene } \\
\text { bridged hybrid (C18, } 50 \times 2.1 \mathrm{~mm} \\
\text { and1.7 } \mu \mathrm{m}) \\
\text { Mobile Phase used: } \\
\text { acetonitrile } \\
\text { formic acid and water } \\
\text { Ratio: (0.1:99.9, v/v) } \\
\text { Flow rate: } 0.25 \text { ml/min } \\
\text { Mass Spectroscopy detection: } \\
\text { Source of detection: Electro-spray } \\
\text { Ionization } \\
\text { Quantification by: different Reaction } \\
\text { Monitoring mode }\end{array}$ \\
\hline 16 & Rat plasma & $\begin{array}{l}\text { (Xu, B.et al., } \\
2015)^{37}\end{array}$ & $\begin{array}{l}\text { Evaporation of the } 10 \mu \mathrm{l} \text { IS with } \\
\text { working tube } \\
\text { Mixing ratio: } 200 \mu \mathrm{l} \text { with internal } \\
\text { evaporated solution } \\
\text { Vortex time: } 1 \mathrm{~min} \\
\text { Mixing ratio: } 10 \mu \mathrm{l} \text { with } 1 \% \text { formic } \\
\text { acid or } 200 \mu \mathrm{l} \\
\text { acetone } \\
\text { Vortex time: } 2 \text { min } \\
\text { Centrifugation time: } 10 \mathrm{~min}, \mathrm{rpm}: \\
\text { 10.000 rpm } \\
\text { Mixing ratio: supernatant by } 200 \mu \mathrm{l} \\
\text { methanol } \\
\text { Vortex time: } 1 \text { min centrifugation } \\
\text { time: } 10 \text { min } \\
\text { Mixing ratio: supernatant by } 400 \mu \mathrm{l} \\
\text { acetonitrile } \\
\text { Vortex time: } 1 \text { min centrifugation } \\
\text { time: } 10 \text { min }\end{array}$ & $\begin{array}{l}\text { Method: } \\
\text { Liquid Chromatography } \\
\text { Mass Spectroscopy } \\
\text { Column used: Zorbax Eclipse eXlta } \\
\text { Dense Bonding (C18, } 150 \times 2.1 \mathrm{~mm} \\
\text { and } 3.5 \mu \mathrm{m}) \\
\text { Mobile Phase used: } \\
\text { acetonitrile and water } \\
\text { Ratio: } 1 \% \\
\text { acetic acid and } 0.001 \text { mol/L } \\
\text { ammonium acetate } \\
\text { Flow rate: } 0.2 \text { ml/min } \\
\text { MS detection: } \\
\text { Source: Electro-spray Ionization } \\
\text { Quantification by: Multiple Reactive } \\
\text { Monitoring mode }\end{array}$ \\
\hline
\end{tabular}




\begin{tabular}{|c|c|c|c|c|}
\hline & & & $\begin{array}{l}\text { Dryness: Nitrogen stream } \\
\text { Temperature: } 37^{\circ} \mathrm{C} \\
\text { Resolubilize with methanol }\end{array}$ & \\
\hline 17 & Rat plasma & $\begin{array}{l}\text { (Yang, L.et } \\
\text { al.,2017) }\end{array}$ & $\begin{array}{l}\text { Solvent used: Methanol Mixture: } 100 \\
\mu \mathrm{l} \text { sample with internal standard of } \\
10 \mu \mathrm{l} \text { Centrifugation time: } 10 \mathrm{~min} \\
\text { rpm: } 12.000 \mathrm{rpm} \\
\text { Temperature: } 4^{\circ} \mathrm{C} \\
\text { Vortex time- } 1 \mathrm{~min} \\
\text { Evaporation: nitrogen stream } \\
\text { Resolubilization with } 100 \mu \mathrm{l} \text { solvent. }\end{array}$ & $\begin{array}{l}\text { Method: } \\
\text { Ultra Performance Liquid } \\
\text { Chromatography } \\
\text { Mass Spectroscopy } \\
\text { Column used: Acquity } \\
\text { Ultra Performance Liquid } \\
\text { Chromatography } \\
\text { Column used: Ethylene Bridged } \\
\text { Hybrid (C18, } 1.7 \mu \text { m, } 50 \times 2.1 \mathrm{~mm} \text { ). } \\
\text { Mobile Phase used: } \\
\text { Acetonitrile } \\
\text { Flow rate: } 0.4 \text { ml/min } \\
\text { formic acid: water } \\
\text { Ratio: (0.1:99.9 v/v) } \\
\text { Quantification by: Multiple reactive } \\
\text { monitoring mode } \\
\text { MS detection: } \\
\text { Source: Electro-spray Ionization. }\end{array}$ \\
\hline 18 & $\begin{array}{l}\text { Stem bark } \\
\text { Mahonia } \\
\text { manipurensis }\end{array}$ & $\begin{array}{l}\text { (Pfoze, N.L. et } \\
\text { al., 2014) }\end{array}$ & $\begin{array}{l}\text { Method: Cold extraction process } \\
\text { Solvent used: Methanol } \\
\text { Quantity: } 1000 \mathrm{~mL} \\
\text { Stirring: room temperature }\end{array}$ & 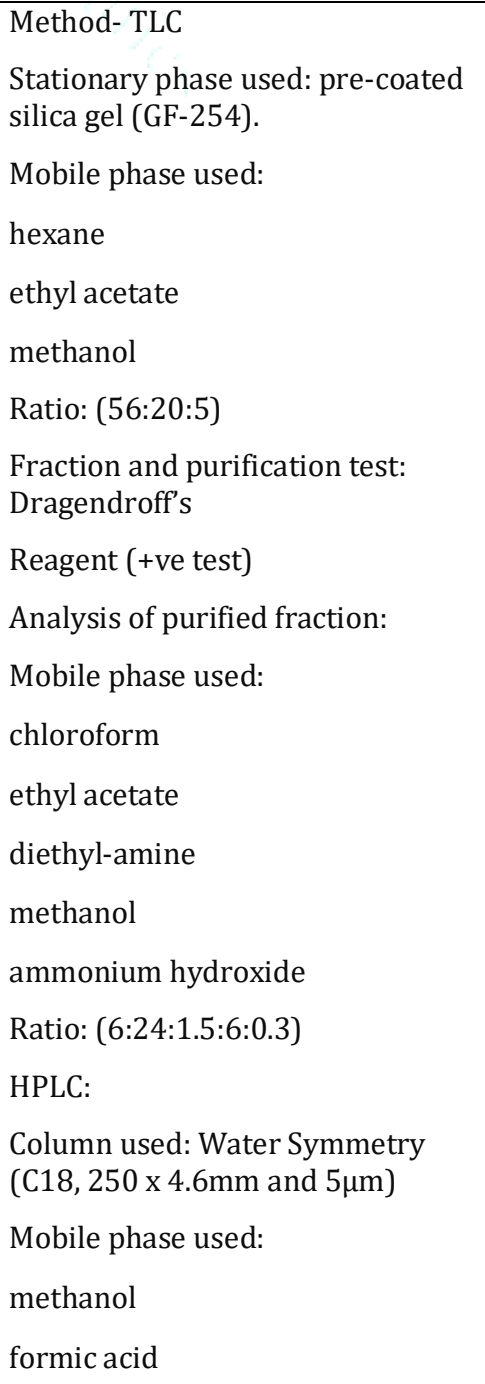 \\
\hline
\end{tabular}




\begin{tabular}{|c|c|c|c|c|}
\hline & & & & $\begin{array}{l}\text { buffer } \\
\text { Ratio: }(0.1 \%, \mathrm{v} / \mathrm{v}) \\
\text { Detection at: } 346 \mathrm{~nm} \\
\text { Flow rate: } 1 \mathrm{ml} / \mathrm{min} \\
\text { Detection by: } \\
\text { UV spectra at } 200 \text { to } 500 \mathrm{~nm} \\
\text { MS: Electro-spray Ionization }\end{array}$ \\
\hline 19 & $\begin{array}{l}\text { Tinospora } \\
\text { cordifolia }\end{array}$ & $\begin{array}{l}\text { (Satija, R.et } \\
\text { al.,2015) }\end{array}$ & $\begin{array}{l}\text { Method: Microwave assisted } \\
\text { extraction (MAE) process } \\
\text { Solvent used: ethanol } \\
\text { Time: } 3 \text { min } \\
\text { Method: Soxhlet extraction process } \\
\text { Solvent used: ethanol } \\
\text { Time: } 3 \mathrm{hr} \\
\text { Filtration } \\
\text { Concentration } \\
\text { Maceration } \\
\text { Solvents for extraction: ethanol- } \\
\text { 200ml by } 7 \text { days irregular stirring }\end{array}$ & $\begin{array}{l}\text { Method: HPTLC } \\
\text { Mobile phase used: } \\
\text { methanol } \\
\text { acetic acid } \\
\text { water } \\
\text { Ratio: (8: } 1: 1 \mathrm{v} / \mathrm{v} / \mathrm{v}) \text {. } \\
\text { Detection at: } 366 \mathrm{~nm}\end{array}$ \\
\hline 20 & $\begin{array}{l}\text { Tinospora } \\
\text { cordifolia, } \\
\text { Tribulus } \\
\text { Terrestris, } \\
\text { Emblica } \\
\text { officinalis }\end{array}$ & $\begin{array}{l}\text { (Joshi, H.et al., } \\
\text { and Kanaki, N.et } \\
\text { al., 2013) }\end{array}$ & $\begin{array}{l}\text { Solvent used for extraction: } \\
\text { chloroform } \\
\text { Sample is triturated with ammonia } \\
\text { solution } \\
\text { Dry: room temperature } \\
\text { Extraction time: } 1 \mathrm{hr} \\
\text { phase extraction of chloroform with } \\
5 \% \text { sulfuric acid } \\
\text { Basic nature: by acid extract with } \\
\text { sodium carbonate } \\
\text { pH: } 9 \\
\text { Extraction with chloroform: } \mathrm{X} 3 \\
\text { Evaporation temperature: } 50^{\circ} \mathrm{C} \\
\text { Residue solubilized by methanol }\end{array}$ & $\begin{array}{l}\text { UV absorbance at: } 348 \mathrm{~nm} \\
\text { (wavelength) }\end{array}$ \\
\hline
\end{tabular}

The extraction process is simple and involves several steps such as sample mixing and extraction solvents e.g. acetone, acetonitrile and methanol. Centrifugation after that the supernatant in nitrogen stream and the extraction of solid phase extraction (SPE) can be applied.

\section{ANALYTICAL TECHNIQUES}

After Berberine extraction, separation, purification and quantification use the chromatography methods. Determination of Berberine extract by using different analytic techniques such as UV-Vis spectrophotometry, High Performance Liquid Chromatography, Thin Layer Chromatography, Capillary Electrophoresis and High Performance Thin Layer Chromatography. Berberine analyzed by Liquid Chromatography-Mass Spectrophotometry, Ultra Performance Liquid Chromatography-Mass Spectrophotometry.

\section{UV-Vis spectrophotometry}

(Joshi, H.et al., 2013; Kanaki, N.et al., 2013), they reported that they work done by this method was considered most effective and rapid detection method from Berberine extract quantitative analysis plant extracts. Berberine concentration determined by UV technique absorption at $348 \mathrm{~nm}$, based on Beer-Lambert Law. Done dilution range of $2-20 \mu \mathrm{g} / \mathrm{ml}$ Berberine samples and compounds avoided by isolation of alkaloid fraction. ${ }^{41}$

\section{High-performance liquid chromatography (HPLC)}

(Sasidharan, S.et al., 2011), reported that HPLC technique widely used for quantitative analysis and qualitative analysis and also used in quantification and identification The stationary phase of C18 silica column and mobile phase used different solvents are methanol, acetonitrile and water with combination phosphate buffers. Separation and detection of Berberine compound by using isocratic gradient elution and 
high sensitivity Berberine identification using UV and DAD techniques. ${ }^{42}$

\section{Thin layer chromatography (TLC) and high performance} thin layer chromatography (HPTLC)

(Samal, P.K.et al., 2013) they reported that two techniques used usually for Berberine detection by TLC method and HPTLC method. These methods were easy and cost effective. By the HPLC method present the chance of running samples at the same time use small quantity of samples as well as mobile phases. The plant Argemone mexicana used for the identification of Berberine by using stationary phase and mobile phase. The silica gel used as stationary phase and mobile phase used with toluene and ethyl acetate solvents. ${ }^{27}$

\section{Electrochemiluminescence (ECL) end-column with CE}

(Du, J.X.et al., and Wang, M.et al., 2010) they reported that used same principles method for detection of Berberine Rhizoma coptidis. They developed the method to identified small volume sample and proved very sensitive with good revolution for Berberine detection. ${ }^{43}$

\section{Mass spectrometry:}

(Xu, B.et al., 2015)they reported that considered great techniques to analyzed samples. In this method, sample analyzed fast and accurate information related to the structural compounds composition in this technique. They developed accurate and sensitive methods to Berberine determine. Samples separation optimized by using six types of reverse phase columns, two mobile phases with different solvents such as methanol-water and acetonitrile-water, additives used in different concentration : $0.1,0.5,1$, and $2 \%$ formic acetic acid and 0.0001, 0.001, $0.01 \mathrm{~mol} / \mathrm{L}$ ammonium acetate, acetic acid and formic acid. The different method was tested in specificity, linearity, lower limit of quantification, stability, accuracy and precision. ${ }^{37}$

\section{POTENTIAL OF BERBERINE ON THEIR THERAPEUTIC EFFECTS}

\section{Immunomodulatory potential:}

Immunomodulatory effect of Berberine was established in many experimental. Berberine furnish to relieve damage in cardiac the by increasing the anti-cardiac myosin antibodies and regulate the action of some STATs or block the differentiation of Th1cytokines and Th2 cytokines cells. This route plays main function with the pathogenesis of myocarditis. Neurologic disease characterized by autoimmune damage peripheral nervous system. The useful outcome of Berberine on animal model resided in its pressure on cellular immunity and humoral immunity and the inhibition of lymphocyte proliferation (CD4) and also decrease in proinflammatory cytokines (IL-6 and TNF). Multiple sclerosis is a common disease of central nervous system and inflammatory processes. Berberine enhances the level of corticosteroids. We can examine the increasing level with the help of experimentally-induced colitis in rats. The benefits and the effects of Berberine may be allow and apply to the rise in level of endogenous glucocorticoid compounds with the effective and desired therapeutic action in inflammatory bowel disease. ${ }^{44-46}$

The various effects of Berberine are shown with their therapeutic and mechanism in Figure 2.

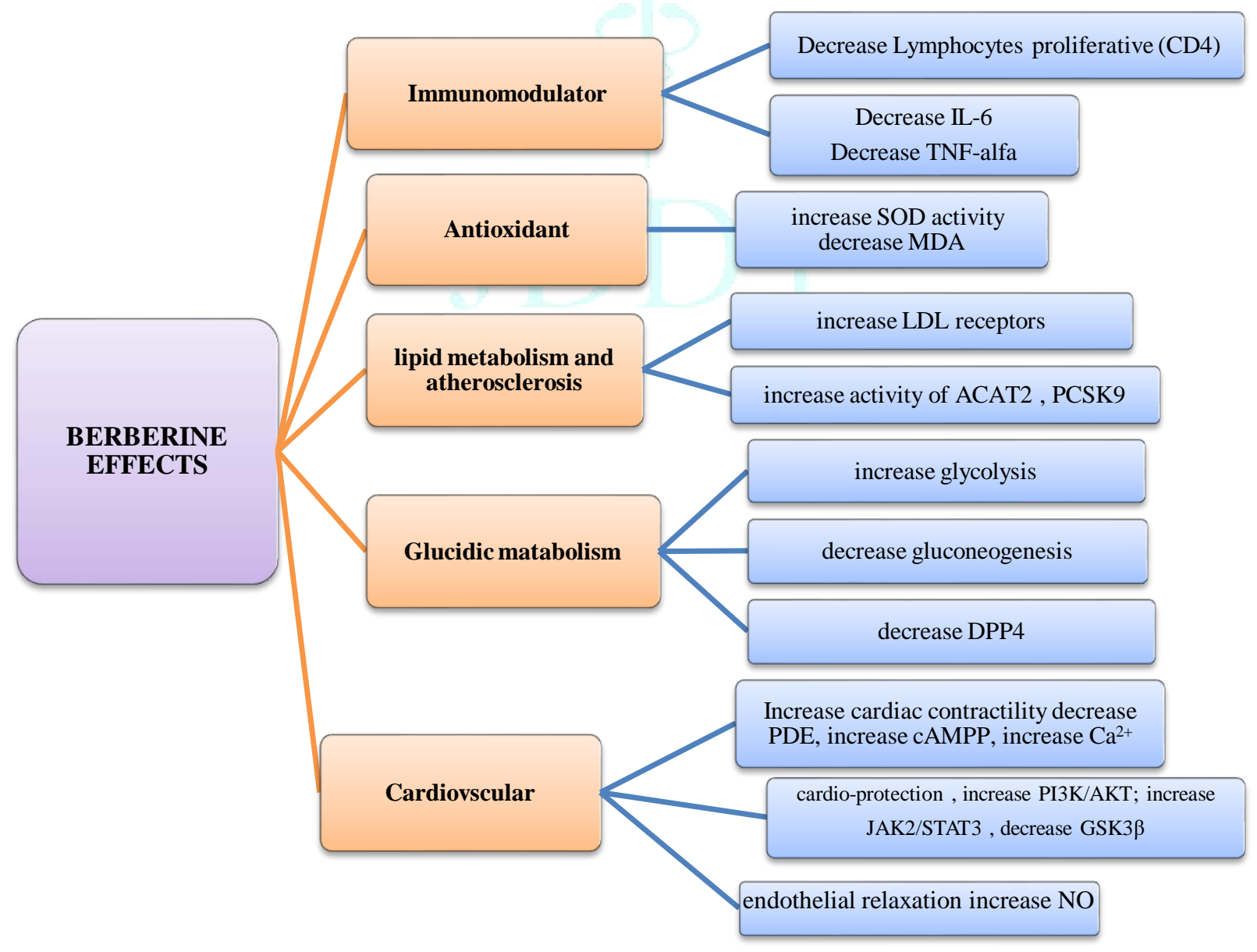

Figure 2: Potential of Berberine on their therapeutic effects and their mechanisms 


\section{Antioxidant potential:}

Normal conditions body maintains balanced the antioxidant and prooxidant agents. Imbalance between pro-oxidant and antioxidant occurs high oxidative stress. Oxidative stress build several mechanisms: a production increase of reactive species, decrease enzyme levels involved in blocking actions of compounds or decrease free radical. The Berberine on peroxidation of lipids, and effect induced after chemical carcinogenesis in small animals. Berberine detects best result of antioxidant properties suitable effect on lipid peroxidation Further, involved mechanism in antioxidant of Berberine: free oxygen removal, ROS/RNS scavenging, nitric oxide ions and reducing destructiveness of superoxide ions. Increase the antioxidant effect of endogenous substances. Highly potential antioxidant and Berberine compared with vitamin C. The oxidative stress plays a main part. ${ }^{47-51}$

\section{Potential on endothelium:}

Berberine induces endothelial relaxation with increasing NO production from essential amino acid through activity of endothelial nitric oxide that thought of within the dilatation method. Berberine facilitates phosphorylation of endothelial nitric oxide synthase and heat shock proteins 90 , that increasing NO production. Endothelial contraction by taking Berberine and it reduced the COX-2 expression. Imbalance of the COX-1 and COX-2 activity, quantitative relation ratio between vasodilator/vasoconstrictor, and prothrombotic/antithrombotic effects. Berberine shows helpful impact on TNFa/AKT/eNOS mRNA beneficial effects. ${ }^{52}$

\section{Atherosclerotic potential:}

The level of blood lipid very high levels and associated with vascular wall swollen. Effect of Berberine during lipid metabolism is the significance of cholesterol low density lipoprotein receptors. Receptors stabilize by an extracellular signal regulated kinase extracellular signal regulated dependent pathway with Berberine increases the activity of low density lipoprotein receptors through JNK pathway. There are two types of enzymes; Acetyl-Coenzyme A Transferase 1 and AcetylCoenzyme A Transferase 2 Berberine influence on lipid profile. 53

\section{Hepatoprotective potential:}

Berberine was established on animal studyof Berberine. Berberine reduced functional hepatic tests and histological damage (cellular infiltrate inflammation and hepatocyte necrosis). The mechanism of Berberine which reduces hepatotoxicity also considered on carbon tetrachloride $\left(\mathrm{CCl}_{4}\right)$ induced hepato-toxicity. Berberine used lowers nitrosamine oxidative stress and inflamed liver. The Berberine decreases. Berberine prevents decrease in increase in lipid peroxidation and super peroxide dismutase activity. Reduction in COX-2, TNF- $\alpha$ and caused nitric oxide synthase (iNOS) levels. Decrease in transaminase levels maintain. Berberine helps to maintain integrity of hepatocellular membrane. ${ }^{54-56}$

\section{Potential on glucose metabolism:}

Berberine low blood sugar level, and its mechanisms Inhibition mitochondrial glucose oxidation. Incentive of glycolysis and breakdown of glucose increased and decreased ATP level through inhibition of mitochondrial function in liver and gluconeogenesis. By the Berberine Inhibition of dipeptidyl peptidase-4 (DPP4), protease responsible for certain peptides such as glucagon-like peptide-1, gastric inhibitory polypeptide; rise insulin level of hyperglycemia, DPP4 inhibition. Determine peptides duration action, so improving glucose tolerance. Berberine improves the insulin resistance, and showed beneficial effects. Hypoglycemic drugs are commonly used and utilization of glucose levels in tissues with helps lowering plasma lipid free fatty acids. ${ }^{57}$

\section{CONCLUSION}

Herbals are the best for the used for the treatment of various diseases with lesser side effects as compared to other formulation. In this review, we came to know about the potential uses of Berberine in the various disorders and in the treatment of various diseases. With the literature, we came to know about that extraction, isolation and analysis of this plant is easy and can be done. So we can prepare the various formulations as herbal formulations for the treatment of various disorders in future.

\section{ACKNOWLEDGEMENT}

The author wishes to acknowledge Laureate Institute of Pharmacy, Jawalamukhi, Himachal Pradesh (176031), for providing their support and other required facilities in the preparation of this review article.

\section{Conflicts of Interest: None}

\section{Funding Support: None}

\section{REFERENCES}

1. Jindal S, Awasthi R, Singhare D, Kulkarni GT, "Topical delivery of Tacrolimus using liposome containing gel: An emerging and synergistic approach in management of psoriasis" Medical Hypotheses, 2020:109838.

2. Seethapathy GS, Raclariu-Manolica AC, Anmarkrud JA Wangensteen H, De Boer HJ. DNA metabarcoding authentication of Ayurvedic herbal products on the European market raises concerns of quality and fidelity. Frontiers in plant science. 2019; 10:68.

3. Devarbhavi $\mathrm{H}$, "Aurvedic and herbal medicine-induced liver injury: It is time to wake up and take notice" springer, 2018;5-7

4. Cui H, Cai Y, Wang L, Jia B, Li J, Zhao S, Chu X, Lin J, Zhang X, Bian Y, Zhuang P. Berberine regulates Treg/Th17 balance to treat ulcerative colitis through modulating the gut microbiota in the colon. Frontiers in pharmacology. 2018; 31(9):571.

5. Cao M, Wang P, Sun C, He W, Wang F. Amelioration of IFN- $\gamma$ and TNF- $\alpha$-induced intestinal epithelial barrier dysfunction by berberine via suppression of MLCK-MLC phosphorylation signaling pathway. PloS one. 2013; 8(5):e61944.

6. Karimov A, "Berberis alkaloids" Chemistry of natural compounds, 1993; 29(4):415-38.

7. Birdsall TC, "Berberine: Therapeutic potential of alkaloid found in several medicinal plants" Altern. Med. Rev, 1997; 2:94-103.

8. Gupta A, "Vitamin D deficiency in India: prevalence, causalities and interventions" Nutrients, 2014; 6(2):729-75.

9. Tandon R, Khanna RD, Dorababu M, Goel RK. Oxidative stress and antioxidants status in peptic ulcer and gastric carcinoma. Indian Journal of Physiology and Pharmacology. 2004 24; 48(1):115-8.

10. Singh A, Duggal S, Singh H, Singh J, Katekhaye S. Withanolides: Phytoconstituents with significant pharmacological activities. International Journal of Green Pharmacy (IJGP). 2010; 4(4).

11. Amritpal S, Sanjiv D, Navpreet K, Jaswinder S, "Berberine: alkaloid with wide spectrum of pharmacological activities" Journal of Natural Products (India), 2010; 3:64-75.

12. Kulkarni SK, Dhir A. Berberine: a plant alkaloid with therapeutic potential for central nervous system disorders. Phytotherapy Research: An International Journal Devoted to Pharmacological and Toxicological Evaluation of Natural Product Derivatives. 2010; 24(3):317-24.

13. Marek R, Sečkářová P, Hulová D, Marek J, Dostál J, Sklenář V. Palmatine and berberine isolation artifacts. Journal of natural products. 2003; 66(4):481-6.

14. Grycova L, Dostál J, Marek R, “Quaternary protoberberine alkaloids" Phytochemistry, 2007; 68(2):150-75.

15. Babu NH, Thriveni HN, Vasudeva R, "Influence of drying methods and extraction procedures on the recovery of berberine content in Coscinium fenestratum" J. Nat. Prod. Plant Resour, 2012; 2:540-4.

16. Teng $\mathrm{H}$, Choi Y. Optimum extraction of bioactive alkaloid compounds from Rhizome coptidis (Coptis chinensis Franch.) using response surface methodology. Solvent Extraction Research and Development, Japan. 2013; 20:91-104.

17. Mokgadi J, Turner C, Torto N. Pressurized hot water extraction of alkaloids in Goldenseal. American Journal of Analytical Chemistry. $2013 ; 2013$.

CODEN (USA): JDDTAO 
18. Rojsanga P, Gritsanapan W. Variation of berberine content in coscinium fenestratum stem in Thailand Market. The Mahidol University Journal of Pharmaceutical Sciences. 2005; 32(3-4):6670.

19. Rojsanga P, Gritsanapan W, Suntornsuk L. Determination of berberine content in the stem extracts of Coscinium fenestratum by TLC densitometry. Medical Principles and Practice. 2006; 15(5):373-8

20. Shigwan H, Saklani A, Hamrapurkar PD, Mane T, Bhatt P. HPLC Method Development and Validation for Quantification of Berberine from Berberis aristata and Berberis tinctoria. International Journal of Applied Science and Engineering. 2013; 11(2):203-11.

21. Alupului A, Calinescu I, Lavric V, “Ultrasonic vs. microwave extraction intensification of active principles from medicinal plants" InAIDIC conference series, 2009; 10(9):1-8.

22. Chang $\mathrm{Y}$, "Ultrasonic-assisted extraction of berberine in ionic liquid pharm" Eng. 2014; 33:1-4.

23. Xu K, He G, Qin J, Cheng X, He H, Zhang D, Peng W. High-efficient extraction of principal medicinal components from fresh Phellodendron bark (cortex phellodendri). Saudi journal of biological sciences. 2018; 25(4):811-5.

24. Xi J. Ultrahigh pressure extraction of bioactive compounds from plants - a review. Critical reviews in food science and nutrition. 2017; 57(6):1097-106.

25. Liao G, Liu J, He S, Chen J, Zhang Z, “Optimization for ultrahigh pressure extraction of berberine from Cortex Phellodendri by central composite design-response surface methodology" Journal of medicinal plants research, 2012; 6(23):3963-70.

26. Mustafa, A. and Turner, C., Pressurized liquid extraction as a green approach in food and herbal plants extraction: A review. Analytica chimica acta, 2011; 703(1):8-18.

27. Samal PK. HPTLC analysis of berberine in Argemone mexicana, L. Journal of global trends in pharmaceutical sciences. 2013; $4(2): 1073-6$

28. Singh R, Ray P, Das A, Sharma M. Penetration of antibiotics through Staphylococcus aureus and Staphylococcus epidermidis biofilms. Journal of antimicrobial chemotherapy. 2010; 65(9):1955-8

29. Liu B, Li W, Chang Y, Dong W, Ni L. Extraction of berberine from rhizome of Coptis chinensis Franch using supercritical fluid extraction. Journal of Pharmaceutical and Biomedical Analysis. 2006; 41(3):1056-60.

30. Liu S, Chen Y, Gu L, Li Y, Wang B, Hao J, Zhu H, Li H, Yang S, Kitanaka S. Effects of ultrahigh pressure extraction conditions on yields of berberine and palmatine from cortex Phellodendri amurensis. Analytical Methods. 2013; 5(17):4506-12.

31. Arawwawala LD, Wickramaarachchi WA, "Berberine content in Coscinium fenestratum (Gaertn.) colebr grown in Sri Lanka" Pharmacologia, 2012; 3(12):679-82.

32. Akowuah GA, Okechukwu PN, Chiam NC, "Evaluation of HPLC and spectrophotometric methods for analysis of bioactive constituent berberine in stem extracts of Coscinium fenestratum" Acta Chromatographica, 2014; 26(2):243-54.

33. Rojsanga P, Sukhthankar M, Krisanapun C, Gritsanapan W, Buripakdi Lawson D, Baek SJ. In vitro anti-proliferative activity of alcoholic stem extract of Coscinium fenestratum in human colorectal cancer cells. experimental and therapeutic medicine. 2010; 1(1):181-6.

34. Patel MC. Isolation of berberine from Berberis aristata by an acid dye method and optimization of parameters. Int. J. Pharm. Sci. Rev. Res. 2013; 20(2):187-9.

35. Liu M, Yin X, Ulin-Avila E, Geng B, Zentgraf T, Ju L, Wang F, Zhang X. A graphene-based broadband optical modulator. Nature. 2011; 474(7349):64-7.

36. Wang Z, Bapst V, Heess N, Mnih V, Munos R, Kavukcuoglu K, de Freitas N. Sample efficient actor-critic with experience replay. arXiv preprint arXiv:1611.01224. 2016; 3

37. Xu B, Li P, Zhang G. Comparative pharmacokinetics of puerarin, daidzin, baicalin, glycyrrhizic acid, liquiritin, berberine, palmatine and jateorhizine by liquid chromatography-mass spectrometry after oral administration of Gegenqinlian decoction and active components alignment (ACA) to rats. Journal of Chromatography B. 2015; 15(988):33-44

38. Yang L, Meng X, Yu X, Kuang H. Simultaneous determination of anemoside B4, phellodendrine, berberine, palmatine, obakunone, esculin, esculetin in rat plasma by UPLC-ESI-MS/MS and its application to a comparative pharmacokinetic study in normal and ulcerative colitis rats. Journal of pharmaceutical and biomedical analysis. 2017; 134:43-52.

39. Pfoze NL, Myrboh B, Kumar Y, Rohman MR. Isolation of protoberberine alkaloids from stem bark of Mahonia manipurensis Takeda using RP-HPLC. Journal of Medicinal Plants. 2014; 2(2):48-57.

40. Satija R, Farrell JA, Gennert D, Schier AF, Regev A. Spatial reconstruction of single-cell gene expression data. Nature biotechnology. 2015; 33(5):495-502.

41. Joshi H, Kanaki N, "Quantitative analysis of berberine in an ayurvedic formulation-Rasayana churna by UV spectrophotometry" J. Pharm. Sci. Biosci. Res, 2013; 3:32-4.

42. Sasidharan S, Chen Y, Saravanan D, Sundram KM, Latha LY Extraction, isolation and characterization of bioactive compounds from plants' extracts. African Journal of Traditional, Complementary and Alternative Medicines. 2011; 8(1).

43. Du JX, Wang M, "Capillary Electrophoresis Determination of Berberine in Pharmaceuticals with End-Column Electrochemiluminescence Detection" Journal of the Chinese Chemical Society, 2010; 57(4A):696-700.

44. Minaiyan M, Ghannadi A, Mahzouni P, Jaffari-Shirazi E. Comparative study of Berberis vulgaris fruit extract and berberine chloride effects on acetic acid-induced colitis in rats. Iranian journal of pharmaceutical research: IJPR. 2011; 10(1):97.

45. Ransohoff RM, Hafler DA, Lucchinetti CF. Multiple sclerosis-a quiet revolution. Nature Reviews Neurology. 2015; 11(3):134.

46. Liu X, Zhang X, Ye L, Yuan H. Protective mechanisms of berberine against experimental autoimmune myocarditis in a rat model. Biomedicine \& Pharmacotherapy. 2016; 1(79):222-30.

47. Bhattacharyya A, Chattopadhyay R, Mitra S, Crowe SE, “Oxidative stress: an essential factor in the pathogenesis of gastrointestinal mucosal diseases" Physiological reviews, 2014; 94(2):329-54.

48. Pilch W, Szygula Z, Tyka AK, Palka T, Tyka A, Cison T, Pilch P, Teleglow A. Disturbances in pro-oxidant-antioxidant balance after passive body overheating and after exercise in elevated ambient temperatures in athletes and untrained men. PloS one. 2014; 9(1):e85320.

49. Ho PL, Chow KH, Lai EL, Lo WU, Yeung MK, Chan J, Chan PY, Yuen KY. Extensive dissemination of CTX-M-producing Escherichia coli with multidrug resistance to 'critically important'antibiotics among food animals in Hong Kong, 2008-10. Journal of antimicrobial chemotherapy. 2011; 66(4):765-8.

50. Rahal A, Kumar A, Singh V, Yadav B, Tiwari R, Chakraborty S, Dhama K. Oxidative stress, prooxidants, and antioxidants: the interplay. BioMed research international. 2014

51. Shirwaikar A, Shirwaikar A, Rajendran K, Punitha IS. In vitro antioxidant studies on the benzyl tetra isoquinoline alkaloid berberine. Biological and Pharmaceutical Bulletin. 2006; 29(9):1906-10.

52. Liu L, Liu J, Huang Z, Yu X, Zhang X, Dou D, Huang Y. Berberine improves endothelial function by inhibiting endoplasmic reticulum stress in the carotid arteries of spontaneously hypertensive rats. Biochemical and Biophysical Research Communications. 2015; 458(4):796-801.

53. Zhu L, Zhang D, Zhu H, Zhu J, Weng S, Dong L, Liu T, Hu Y, Shen X. Berberine treatment increases Akkermansia in the gut and improves high-fat diet-induced atherosclerosis in Apoe-/- mice. Atherosclerosis. 2018; 268:117-26.

54. Zhao G, Cawood PA. Precambrian geology of China. Precambrian Research. 2012; 222:13-54.

55. Domitrović R, Jakovac H, Blagojević G, "Hepatoprotective activity of berberine is mediated by inhibition of TNF- $\alpha$, COX-2, and iNOS expression in CCl4-intoxicated mice" Toxicology, 2011; 280(12):33-43.

56. Mehrzadi S, Fatemi I, Esmaeilizadeh M, Ghaznavi H, Kalantar H, Goudarzi M. Hepatoprotective effect of berberine against methotrexate induced liver toxicity in rats. Biomedicine \& Pharmacotherapy. 2018; 97:233-9.

57. Neag MA, Mocan A, Echeverría J, Pop RM, Bocsan CI, Crișan G, Buzoianu AD. Berberine: Botanical occurrence, traditional uses, extraction methods, and relevance in cardiovascular, metabolic, hepatic, and renal disorders. Frontiers in pharmacology. 2018; 21(9):557. 